\title{
Sterols sense swelling in lipid bilayers
}

\author{
Frank Richter, ${ }^{1,2, *}$ Leonard Finegold, ${ }^{3, \dagger}$ and Gert Rapp ${ }^{1, \ddagger}$ \\ ${ }^{1}$ European Molecular Biology Laboratory, Outstation Hamburg, DESY, Notkestraße 85, D-22603 Hamburg, Germany \\ ${ }^{2}$ Department of Physics, E22 Biophysics, TU Munich, D-85748 Garching, Germany \\ ${ }^{3}$ Department of Physics, Drexel University, Philadelphia, Pennsylvania 19104
}

(Received 10 September 1998)

\begin{abstract}
In the mimetic membrane system of phosphatidylcholine bilayers, thickening ("precritical behavior," "anomalous swelling") of the bilayers is observed, in the vicinity of the main transition, which is nonlinear with temperature. The sterols cholesterol and androsten are used as sensors in a time-resolved simultaneous small- and wide angle $\mathrm{x}$-ray diffraction study to investigate the cause of the thickening. We observe precritical behavior in the pure lipid system, as well as with sterol concentrations less than $15 \%$. To describe the precritical behavior, we introduce a theory of precritical phenomena. The good temperature resolution of the data shows that a theory of the influence of fluctuations needs modification. The main cause of the critical behavior appears to be a changing hydration of the bilayer. [S1063-651X(99)10803-1]
\end{abstract}

PACS number(s): 87.16.Dg, 05.70.Jk, 61.10.Eq, 64.60.Fr

\section{INTRODUCTION}

Our ultimate aim is a fuller description of biological cell membranes, via the physics of membrane models. Cell membranes are mainly in equilibrium, yet obviously must experience fluctuating nonequilibrium conditions during cellular events such as membrane fusion and transmembrane signaling. An excellent experimental model system for biological cell membranes is a dispersion of phospholipid in water, known as liposomes, which exhibit a variety of interesting phases as temperature is changed [1]. Major changes in physical properties are seen at the gel to fluid phase transition, the main transition at temperature $T_{m}$, which is a firstorder transition [2,3], occurring over a narrow temperature range. However, when approaching the main transition from above $T_{m}$, structure-sensitive methods reveal a nonlinear increase (with decreasing temperature) of the lattice parameter $d$ in phosphatidylcholine (PC) membranes [4-6], which is attributed to critical behavior (illustrated in Fig. 5). This increase is here termed "anomalous" behavior. This term has been used also in other contexts [6]. The corresponding critical temperature $T_{c}$ would lie at a temperature just below that of $T_{m}$, and so is "hidden" by the main transition. Critical behavior is also seen in liposomes by nuclear magnetic resonance (NMR) [7] and ultrasonic absorption [8]. This behavior in phosphatidylcholine membranes, a major component of cell membranes, is believed to be of physiological importance, because small temperature changes change the thickness of the membrane drastically and thus can easily affect protein functions [9,10]. Additional support for biological relevance comes from the fact that the nonlinear behavior occurs in the neighborhood of a phase transition, a nonequilibrium state of matter.

The bilayer thickness $d$, measured by x-ray diffraction, is composed of three regions which are the headgroup thick-

\footnotetext{
*Electronic address: frichter@embl-hamburg.de

${ }^{\dagger}$ Electronic address: L@Drexel.edu

*Electronic address: rapp@embl-hamburg.de
}

ness $d_{H}$, which is part of the bilayer thickness $d_{B}$, and the inter-bilayer water layer $d_{W}$, where $d_{B}+d_{W}=d$. The aim is to disentangle the contribution of each region to the observed critical thickening in $d$, by examining four physically different models, each of which affect mainly one region; the models do not necessarily agree [4-6,11]. In brief, model I (following the description of the models by Nagle $[2,40]$ ) attributes the thickening to critical fluctuations, where a softening of the bilayer leads to a reduced bending modulus (via enhanced Helfrich undulations) [12], and affects mainly $d_{W}$ $[13,14]$. This model has been tested and supported by neutron scattering analysis $[6,11]$. An analysis of x-ray data [4] did not confirm the reduced bending modulus. Model II attributes the thickening to an increasing conformational order in the hydrocarbon chains upon approaching $T_{c}$, and hence to an increase in the lipid bilayer thickness $d_{B}$ [4]. Both analyses use mean-field-type liquid crystal theories whose application to critical phenomena and phase transitionsaccording to the Landau theory the $L_{\alpha}-P_{\beta^{\prime}}$ transition has no chance of being second order [15] — is debatable. In model III, the thickening is attributed to interbilayer hydration and van der Waals forces, which increase the water layer thickness $d_{W}$ [2]. Reasons for a change in these forces when approaching the transition and their effect on the bilayer are not well understood, nor is the functional form modeling these forces tested in the neighborhood of the main transition. Model IV attributes the thickening to an increased headgroup layer thickness $d_{H}$ only, which is, however, not supported by NMR work $[16,40]$.

Sterols are an important constituent of eukaryotic membranes. In mammals, cholesterol is essential for the wellbeing of cells [3]; a concentration-dependent effect on softening and stiffening the membrane has been found for cholesterol [11]. Sterols can also play a role in some protein functions coupling lipid dynamics to protein dynamics [3], and in the action of an acetylcholine receptor [17]. Cholesterol organizes submicron domains in living cells [18]. Here, in the absence of proteins, sterols serve us as a natural sensor of the lipid dynamics. In this context it is worthwhile to mention that a high content of cholesterol ( $>20 \mathrm{~mol} \%$ ) 
changes the lipid phase diagram drastically, whereas small amounts $(<10 \mathrm{~mol} \%)$ do not affect the lipid phase diagram significantly [3]. Note that a phase diagram contains information about the equilibrium phases, and that it does not give direct information about nonequilibrium effects which may naturally occur at the phase boundaries. It has been shown [11] that small amounts of cholesterol strongly affect the structure of the lipid membrane near the main transition.

Our first aim in studying the phenomenon of increased swelling is to find out whether it is analytical or indeed anomalous. If the latter is found, i.e., the $d$ spacing close to a transition can be fit to an expression of the form $\left[\left(T-T_{c}\right) / T_{c}\right]^{-\alpha}$, where $T_{c}$ is the critical temperature and $\alpha$ the critical exponent, then we intend to quantify the effect by determining the critical exponent, and test the theoretical predictions. Critically behaving bilayer systems are of interest because of their low dimensionality and the implicit possibility of long-range ordering effects in fluid phases.

The main goal is to study various membrane interactions in order to identify the source of the anomalous swelling in PC membranes. We probe the interactions with two different sterols, cholesterol, and androsten. In terms of structure, cholesterol resembles androsten prolonged by an extra aliphatic tail. The relative sizes and position of the two sterols and the PC bilayer are such that the sterols are about half the length of a lipid molecule, and they lie towards the water interface [3].

\section{EXPERIMENT}

\section{A. Protocol}

Using time-resolved simultaneous small- and wide-angle $\mathrm{x}$-ray diffraction (SAX and WAX), we are able to retrieve structural information in the region about the main transition as well as in the neighboring phases. The experiments were conducted at beam line X13 of EMBL at DESY, Hamburg. Beam line [19] and data acquisition system [20] have been been described in detail. Here, we report measurements on dimyristoylphosphatidylcholine (DMPC), though we have performed analogous experiments on dilauroylphosphatidylcholine (DLPC) and dipalmitoylphosphatidylcholine (DPPC). The DMPC and cholesterol were from Avanti (Alabaster, AL), and androsten (androsten-3 $\beta$-ol) from Steraloids (Wilton, NH). Sample preparation is described elsewhere [3]. A single experiment consisted of a first heating from $4{ }^{\circ} \mathrm{C}$ to $29{ }^{\circ} \mathrm{C}$, i.e., to more than $5{ }^{\circ} \mathrm{C}$ above the main transition temperature. The sample was then cooled to $10{ }^{\circ} \mathrm{C}$, followed by a second heating up to $29{ }^{\circ} \mathrm{C}$; i.e., each cycle consisted of three ramps. This procedure was chosen to exclude effects from improper mixing of lipids and sterols during sample preparation, to single out phase effects caused by a slow kinetics and/or metastability, and to look at possible hysteretic behavior. The scan rate was $0.5^{\circ} \mathrm{C} / \mathrm{min}$ within the phases, and $0.25{ }^{\circ} \mathrm{C} / \mathrm{min}$ between $19{ }^{\circ} \mathrm{C}$ and $26{ }^{\circ} \mathrm{C}$ covering the main transition. This rate was determined in test experiments with the lipid only. A second sample stayed during the experiment in the same sample holder but was not exposed to the beam. It was always measured as a reference, prior to and after the temperature cycle, to check for possible radiation damage.

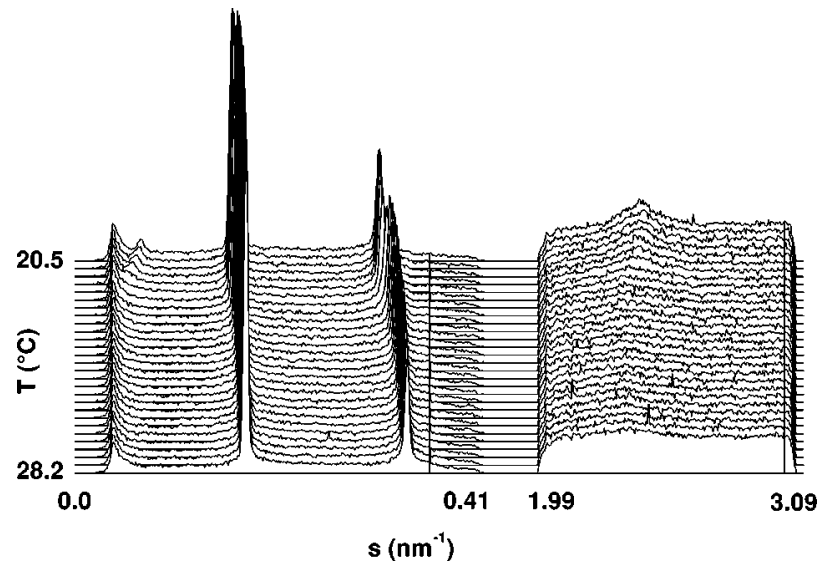

FIG. 1. Small- and wide-angle x-ray diffraction patterns of dimyristoylphosphatidylcholine vesicles with $10 \mathrm{~mol} \%$ androsten. Raw data of the first heating through the main transition are shown. The scattering vector $s=1 / d=(2 \sin \theta) / \lambda$, with $2 \theta$ the Bragg angle.

\section{B. Data}

From our x-ray data we directly determine the positions of the first- and second-order lamellar reflections, as well as the wide-angle reflections due to the ordering of the aliphatic chains. Typical simultaneous small- and wide-angle $\mathrm{x}$-ray diffraction patterns of vesicles of DMPC with $10 \mathrm{~mol} \%$ androsten are shown in Fig. 1.

The swelling is seen by the movement of the layer reflections in the SAX region towards smaller $s$ values (i.e., smaller angles) upon approaching the main transition. The ripple phase is identified by the (10) reflection, which is seen near the beam stop edge, as well as by the rising reflection from the ordering chains in the WAX region.

We recorded two orders of reflections in order to better determine the position of the reflections since, especially in the ripple phase and in the coexistence region, there is an overlap due to scattering from the in-plane ripple structure. Figure 2 shows the $d$ spacing $d(T)$ for the variety of samples measured.

\section{RESULTS}

\section{A. Determination of $\boldsymbol{T}_{\boldsymbol{m}}$ from x-ray measurements}

If only SAX data are collected for measuring $T_{m}$, then for PC systems the transition temperature $T_{m}^{\mathrm{sax}}$ might be determined as a major change in slope $d d(T) / d T$ at the lowtemperature end of the swelling [2]. In particular, when the $d$ spacing of the two phases participating in the transition is continuously changing, $T_{m}$ cannot be determined with precision. In combined time-resolved SAX and WAX studies, we could define a transition temperature $T_{m}$ at which the intensity of the chain reflection recorded in the ripple phase is reduced to half of its maximum. Instead, taking into account the coexistence of the ripple phase and the $L_{\alpha}$ phase, we here define a transition zone $\Delta T_{m}^{\mathrm{wax}}$ over which the intensity drops with increasing temperature. Though the WAX measurements describe the chain melting process characterizing $T_{m}$ (and the degree of chain ordering could serve as order parameter), these measurements depend-to a certain degree-on long-range periodic order among the chains. 


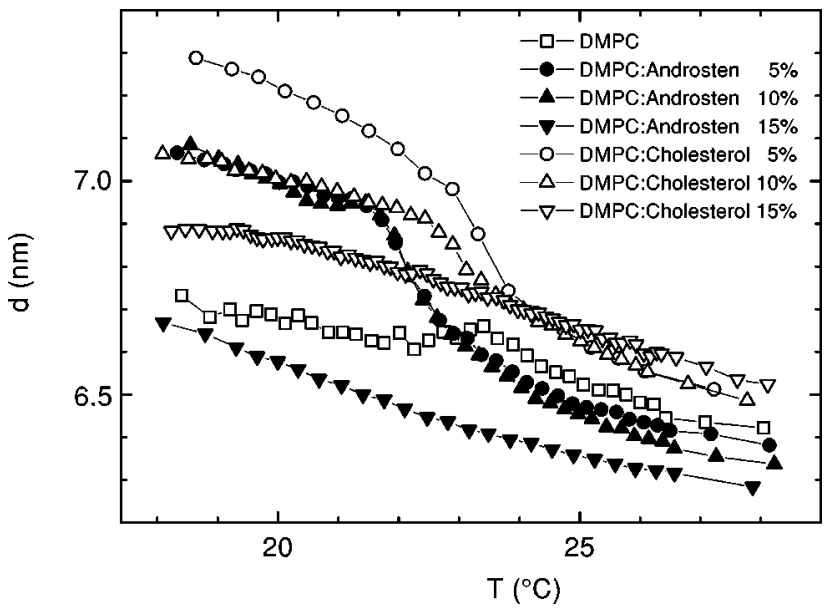

FIG. 2. Effects of cholesterol and of androsten on the bilayer thickness $d$ of dimyristoylphosphatidylcholine vesicles: results of the first heating (see text) are shown. The experimental points are connected by lines to aid the eye.

Moreover, it is by no means obvious that the interactions responsible for the chain ordering are directly related to the critical bilayer swelling $d$. One requires, in addition, a relation between the cooperativity of chain ordering and critical behavior.

The $d$ spacing-as well as the intensity of the ripple reflection-exhibits a very strong temperature dependence near the main transition, as already studied in detail [21], which is evidence of the nonequilibrium state of the ripple phase at least near the main transition. The formation of the ripple phase itself might thus be a critical phenomenon, too. The existence of the ripple phase appears to be linked to the processes on the higher temperature side of the main transition. We also note that of all the samples being considered for anomalous behavior, only those displaying a local maximum in $d(T)$ at the main transition show a clear (10) ripple reflection that forms simultaneously during the main transition (Fig. 3).

\section{B. Is the swelling critical?}

\section{Critical behavior}

Any singular behavior (power-law- or logarithmicdivergent, as well as a finite peak) of a quantity $f(T)$ about a critical temperature $T_{c}$, can be embraced in one single functional formula [22]:

$$
f(T) \sim \frac{1}{\alpha}\left(\left|\frac{\left(T-T_{c}\right)}{T_{c}}\right|^{-\alpha}-1\right),
$$

where $\alpha$ is the critical exponent. We preserve the name $\alpha$ to point out the similarity of the lattice swelling to the increasing specific heat (with decreasing temperature) in the transition. In a $\log -\log$ representation of $f(T)$, a fit then yields the critical parameters $\alpha$ and $T_{c}$. If a nonlinear-yet well behaved-graph of the log-log data is obtained, the function $f(T)$ can be described analytically. Therefore, the representation of formula (1) serves as a test to distinguish between analytical and critical behavior. A critical exponent of zero needs some further investigation, since it either indicates a

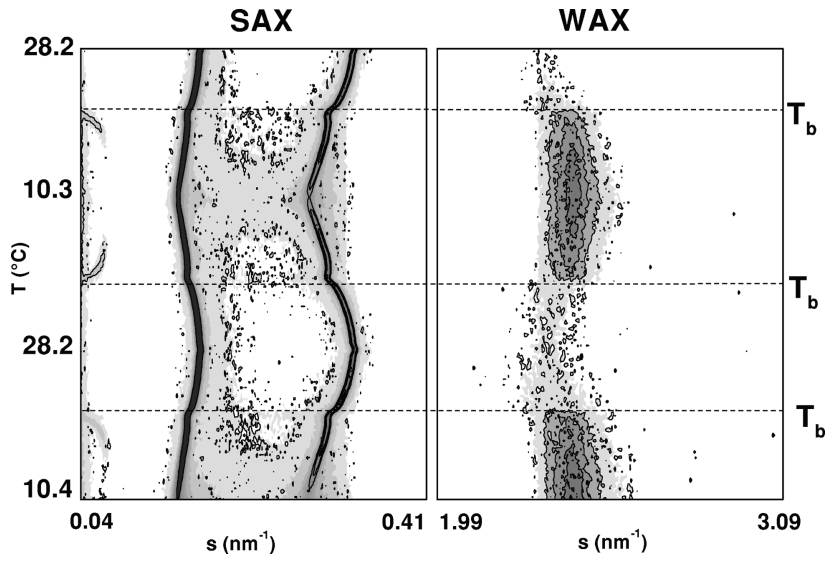

FIG. 3. Contour plot of the intensities recorded in the $10 \mathrm{~mol} \%$ androsten (in dimyristoylphosphatidylcholine vesicles) experiment as a function of the scattering vector $s$ and temperature $T$ beginning at $10.4{ }^{\circ} \mathrm{C}$. Small- (first two orders seen) and wideangle (chains) x-ray diffraction patterns are shown. $T_{b}$ is defined in the text at Table I.

logarithmic divergence, a cusplike behavior, or a perfectly analytical function with no anomalous behavior worse than a jump discontinuity [23]. The log-log plots (Fig. 4) disclose a critical behavior of $d(T)$.

Here we address the problem of determining $T_{c}$ for a precritical system, i.e., where $T_{c}$ is hidden by the phase transition. Formally, this problem is implicit to the definition of a critical exponent [formula (1)] which implies the knowledge of $T_{c}$.

\section{Determination of $T_{c}$}

When a precritical system is in the swelling region where it displays critical behavior, it does not "know" that it will, upon approaching $T_{c}$, undergo a first-order transition. So we can treat the precritical system over the swelling region as a critical system $[7,8]$. The fact that the system does not "know" a priori the order of the transition it will undergo when it swells critically is the base for the theory of critical unbinding of membranes [14], which means the $d$ spacing can diverge. Therefore, we can reduce formula (1) to the case $\alpha>0$ [22].

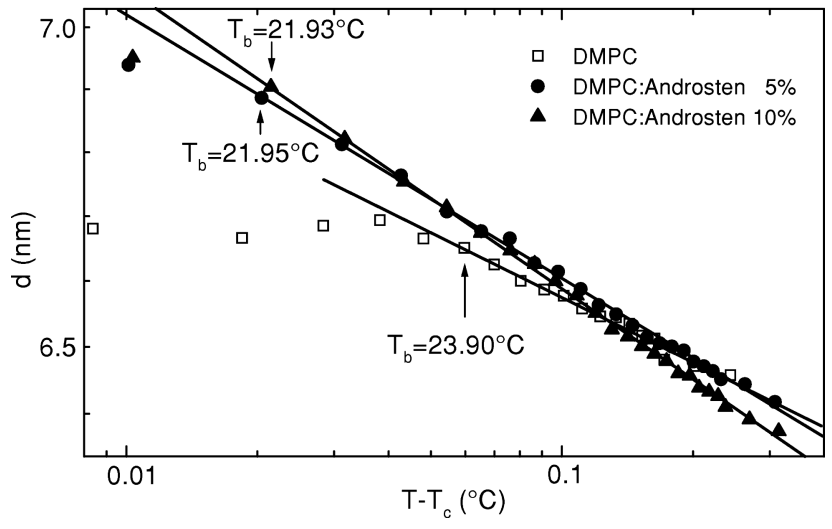

FIG. 4. Anomalous swelling: log-log plot of the bilayer thickness $d$ (of vesicles of dimyristoylphosphatidylcholine with 0,5 , or $10 \mathrm{~mol} \%$ of androsten) is fitted to expression (2). Linear fits yield $\alpha=0.01175,0.02715$ and 0.03074 , respectively (first heating, see text and Table I). The last point on the line is labeled $T_{b}$. 


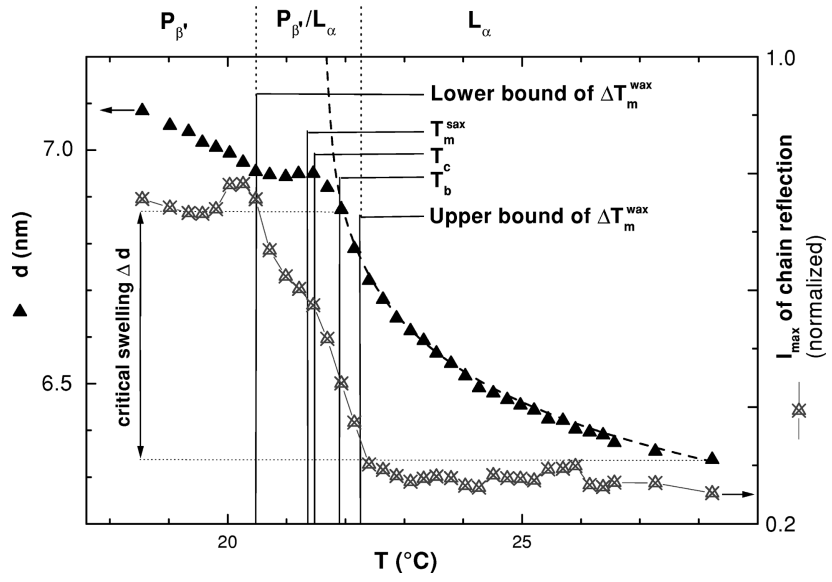

FIG. 5. Illustration of transition-related temperatures: lattice spacing $d$ versus temperature (upper graph, closed symbols) measured for dimyristoylphosphatidylcholine vesicles with $10 \mathrm{~mol} \%$ androsten during the first heating. The dashed line is a fit to expression (2). The lower graph shows the maximum intensity of the chain reflection (normalized to the intensity measured at $T$ $=10{ }^{\circ} \mathrm{C}$ ). The drop in intensity indicates the melting of the chain lattice during the main transition.

$$
\ln d(T) \sim \ln \left(\frac{\left(T-T_{c}\right)}{T_{c}}\right) \quad \text { or } \quad d(T) \sim\left(\frac{\left(T-T_{c}\right)}{T_{c}}\right)^{-\alpha}
$$

In the following, we use Fig. 4 (DMPC, first heating) as an example. To obtain $T_{c}$ we employ a least-squares minimization technique while varying $T_{c}$. If the system behaves critically, then all data points (starting from the highest temperature data point $T_{i}$ ) will follow a linear function in the log-log plot, upon appropriate choice of $T_{c}$. The advantage of this method is that, beginning at the highest temperatures $T_{i}$ (for this example $T_{i}=28.05{ }^{\circ} \mathrm{C}$ ), it first finds the maximum number of data points $b$ that could follow a linear function, by comparing the measured $d$ spacings under the constraint $d\left(T_{i}\right)-d\left(T_{i+1}\right)<d\left(T_{i+1}\right)-d\left(T_{i+2}\right), i=\{1, b\}$. The temperature recorded at the data point at the low-temperature end $\left(T_{b}=23.90{ }^{\circ} \mathrm{C}\right)$ of the thus-defined region of anomalous swelling gives the starting temperature of the $T_{c}$ variation. The system behaves critically if all the data points in the anomalous swelling region indeed make a linear function in the log-log-representation; if not, the swelling is not critical. In the next step, if the data follow a linear function, the slope of this linear function (i.e., the critical exponent) is found by minimizing the sum of squares of deviations from the line. All of the data points enter with the same weight. The first data point outside the purely critical regime (at $23.60{ }^{\circ} \mathrm{C}$ ) is easily distinguished, even by visual inspection, by its large deviation off the linear fit in the log-log representation (Fig. 4). The preceding nondeviating point, defining the "breakdown temperature"' $T_{b}$, where the critical behavior breaks down, is at $23.90{ }^{\circ} \mathrm{C}$ in Fig. 4. The existence of such a data point follows from the observation of precritical phenomena. The procedure finds the global minimum because raising or lowering of $T_{c}$ yields a systematic concave or convex distortion, respectively, of the string of data points from a straight line.

Table I gives $T_{c}$, the temperature $T_{b}$ of the last nondeviating data point in the critical regime closest to $T_{c}$, and the critical exponent $\alpha$, along with the transition temperatures $T_{m}^{\mathrm{sax}}$ obtained from the $d(T)$ plot, and $\Delta T_{m}^{\mathrm{wax}}$, as well as the amount of anomalous swelling observed $\left[d\left(T_{b}\right)\right.$ $\left.-d\left(28{ }^{\circ} \mathrm{C}\right)\right]$. These temperatures are illustrated in Fig. 5.

The critical behavior is observed (Fig. 4) in the region closest to the critical point over at least a full decade in $(T$ $\left.-T_{c}\right) / T_{c}$, where data have been collected. For all of the samples listed, the single value of $T_{c}$ itself is to be interpreted with caution. We remind ourselves that we do not directly look at the critical behavior about a second-order transition point $T_{c}$. Instead, the first-order transition intercedes, and thus eliminates from observation the region in the immediate vicinity of $T_{c}$. This region is the most important for determining the critical exponent for critical phenomena, because there the accompanying long-wavelength fluctuations govern the physical properties of a critical system and have to be taken into account, e.g., by means of RG theory [24].

The advantages of our approach to investigate the criticality of a system are manifold: no $a b$ initio model needs to be assumed, which is a valuable asset especially for critical phenomena and phase transitions; no fitting of experimental data other than the linear fit (to the log-log plots) to determine the exponent is needed; and no adjustable parameters are used. The breakdown temperature $T_{b}$, replacing the nonobservable critical temperature $T_{c}$ when one applies the theory of critical phenomena, is not very sensitive to the fit. On the other hand, $T_{c}$ and $\alpha$ are more sensitive to the fit, also due to the relatively small temperature range where data have been collected. However, the exact value of the critical temperature $T_{c}$, and hence the obtained value of the exponent, is not crucial for the interpretation of precritical phenomena. We stress that it is the breakdown temperature $T_{b}$, and not the critical temperature $T_{c}$, that is characteristic for precritical phenomena. For critical phenomena $T_{b}=T_{c}$. In our experiments we find in all cases $T_{b}>T_{c}$.

\section{The precritical parameters}

The exponents derived are all close to zero, and in light of the theory of critical phenomena, a small magnitude of an exponent implies a sharp divergence [23]. The exponent $\alpha$ is the quantity that allows us to rank precritical phenomena. With one exception $\alpha$ is always smaller for the pure lipid than for the sterol samples (Table I). Following the theory for critical phenomena, this implies that the $d(T)$ dependence for the pure lipid is the closest to criticality, i.e., the pure lipid would display a singularity of a relatively higher order at $T_{c}$ than would the sterol samples. Indeed, looking at the $d(T)$ plots obtained from the layer reflections (Fig. 2), the pure lipid shows the flattest region between $28{ }^{\circ} \mathrm{C}$ and $T_{m}^{\mathrm{sax}}$ (at $\sim 23.5{ }^{\circ} \mathrm{C}$ ). We conclude that the higher the concentration of sterol (for those samples that display critical behavior), the higher is $\alpha$, i.e., the less critical is the behavior. This is related to the breakdown of criticality further away from $T_{c}$.

It is further noticed, for samples showing the strongest swelling effect in Fig. 2 [i.e., $d\left(T_{b}\right)-d\left(28{ }^{\circ} \mathrm{C}\right)$ for $5 \mathrm{~mol} \%$ cholesterol and $10 \mathrm{~mol} \%$ androsten], that the two temperatures obtained in our analysis of the precritical behavior $\left(T_{c}\right.$ and $\left.T_{b}\right)$ are relatively close (Table I). Then, 
TABLE I. Critical parameters, $T_{c}$ (critical temperature), $T_{b}$ (breakdown temperature), and $\alpha$ (exponent), for dimyristoylphosphatidylcholine vesicles with two sterols at various concentrations (conc.). Also given are the main transition temperatures from small- and wide-angle x-ray diffraction $T_{m}^{\mathrm{sax}}, \Delta T_{m}^{\mathrm{wax}}$, and the anomalous swelling $d\left(T_{b}\right)-d\left(28{ }^{\circ} \mathrm{C}\right)$ (illustrated in Fig. 5).

\begin{tabular}{|c|c|c|c|c|c|c|c|c|}
\hline Sterol & $\begin{array}{c}\text { Conc. } \\
(\mathrm{mol} \mathrm{\%})\end{array}$ & $\begin{array}{l}T_{m}^{\mathrm{sax}} \\
\left({ }^{\circ} \mathrm{C}\right)\end{array}$ & $\begin{array}{c}\Delta T_{m}^{\mathrm{wax}} \\
\left({ }^{\circ} \mathrm{C}\right)\end{array}$ & $\begin{array}{c}T_{c} \\
\left({ }^{\circ} \mathrm{C}\right)\end{array}$ & $\begin{array}{c}T_{b} \\
\left({ }^{\circ} \mathrm{C}\right)\end{array}$ & $\begin{array}{l}T_{b}-T_{c} \\
\quad\left({ }^{\circ} \mathrm{C}\right)\end{array}$ & $\alpha$ & $\begin{array}{c}d\left(T_{b}\right)-d\left(28{ }^{\circ} \mathrm{C}\right) \\
(\mathrm{nm})\end{array}$ \\
\hline \multicolumn{9}{|l|}{ 1st Heating: } \\
\hline None & 0 & 23.3 & $23.3-23.9$ & 22.55 & 23.9 & 1.4 & 0.02175 & 0.24 \\
\hline Androsten & 5 & 21.2 & $21.0-22.3$ & 21.51 & 21.9 & 0.4 & 0.02715 & 0.47 \\
\hline Androsten & 10 & 21.3 & $20.5-22.2$ & 21.47 & 21.9 & 0.4 & 0.03074 & 0.54 \\
\hline Cholesterol & 5 & 22.6 & & 23.03 & 23.3 & 0.3 & 0.01771 & 0.39 \\
\hline Cholesterol & 10 & 22.4 & $21.9-23.0$ & 21.24 & 22.4 & 1.2 & 0.03827 & 0.42 \\
\hline \multicolumn{9}{|l|}{ 1st Cooling: } \\
\hline None & 0 & 23.0 & $23.6-22.8$ & 22.45 & 23.7 & 1.3 & 0.02512 & 0.27 \\
\hline Androsten & 5 & 21.0 & $22.0-19.9$ & 20.90 & 22.1 & 1.2 & 0.03796 & 0.42 \\
\hline Androsten & 10 & 21.0 & $21.8-21.0$ & 21.06 & 22.1 & 1.0 & 0.03409 & 0.39 \\
\hline Cholesterol & 5 & 22.2 & & 21.86 & 22.5 & 0.6 & 0.02714 & 0.44 \\
\hline Cholesterol & 10 & 22.1 & $22.4-22.0$ & 19.72 & 22.2 & 2.5 & 0.05164 & 0.40 \\
\hline \multicolumn{9}{|l|}{ 2nd Heating: } \\
\hline None & 0 & 23.2 & $23.2-23.7$ & 22.89 & 23.9 & 1.0 & 0.02264 & 0.30 \\
\hline Androsten & 5 & & $20.0-22.0$ & 21.28 & 22.1 & 0.8 & 0.03445 & 0.46 \\
\hline Androsten & 10 & 21.3 & $21.2-22.3$ & 21.40 & 21.9 & 0.5 & 0.03329 & 0.55 \\
\hline Cholesterol & 5 & 23.0 & $22.8-23.4$ & 22.72 & 23.0 & 0.3 & 0.02453 & 0.40 \\
\hline Cholesterol & 10 & 22.3 & $21.8-22.9$ & 20.90 & 22.7 & 1.8 & 0.04173 & 0.37 \\
\hline
\end{tabular}

both temperatures have a qualitatively similar dependence on the sterol concentration pointing towards a relationship among each other.

\section{Transition-related temperature correlations}

Table I shows that the lowering of $T_{m}^{\mathrm{sax}}$ depends on the sterol, since androsten lowers $T_{m}^{\mathrm{sax}}$ more than cholesterol. For both sterols the two concentrations tested, $T_{m}^{\mathrm{sax}}$ and $\Delta T_{m}^{\mathrm{wax}}$, do not differ significantly. However, the number of concentrations measured is not enough to conclude that these temperatures are only weakly dependent on the sterol concentration, as long as the system shows the anomalous swelling [11]. There is only a small temperature hysteresis. For $T_{c}$ we see a similar behavior with the exception that there is an obvious dependence on the cholesterol concentration. The dependence of $T_{b}$ on the sterol concentration is analogous to that of $T_{m}^{\mathrm{sax}}$, but the lowering is not as pronounced. $\Delta T_{m}^{\mathrm{wax}}$ features the same tendency as $T_{b}$.

The samples displaying the largest swelling effect [i.e., $\left.d\left(T_{b}\right)-d\left(28{ }^{\circ} \mathrm{C}\right)\right]$, which are the $5 \mathrm{~mol} \%$ cholesterol and the 5 and $10 \mathrm{~mol} \%$ androsten samples (see Table I), yield a lower $T_{m}^{\mathrm{sax}}$ than $T_{c}$. Of course, this does not mean that these samples show a real continuous transition with an observable critical point, since we always find $T_{m}^{\mathrm{sax}}<T_{b}$, i.e., on cooling, the swelling remains precritical and the system never reaches the maximum swelling before it leaves the critical regime. On the other hand, the breakdown of critical behavior at sub-maximal swelling [e.g., for 5 mol $\%$ androsten $\left.d\left(T_{b}\right)=6.86 \mathrm{~nm}<d\left(T_{m}^{\mathrm{sax}}\right)=6.95 \mathrm{~nm}\right]$ (cf. Fig. 5) implies that the systems continue to swell below $T_{b}$ but not in a critical manner. This noncritical swelling, accompanied by the continued chain ordering $\left[T_{b}\right.$ is always larger than both $T_{m}^{\mathrm{sax}}$ and the lower end of the transition zone $\Delta T_{m}^{\mathrm{wax}}$ (Table I)], is evidence that the chain ordering does not obey critical dynamics in the immediate neighborhood of the maximum swelling. On heating, a decreasing chain ordering, combined with a noncritical reduction of the lattice parameter in the vicinity of its maximum, is seen. $T_{b}$ is again always larger than $T_{m}^{\mathrm{sax}}$ and the lower end of the transition zone $\Delta T_{m}^{\text {wax }}$ (Table I). Therefore, only at temperatures $T_{b}$ higher than $T_{m}^{\mathrm{sax}}$ does the system move into the critical regime (Table I). For all samples, we generally find $T_{m}^{\mathrm{sax}}$ on the lower temperature side of the transition zone $\Delta T_{m}^{\text {wax }}$ (Table I). Thus, on heating, the $d$ spacing does not start to decrease before there is a decrease in the chain ordering. On cooling, the swelling does not stop before there is already periodic chain ordering established. This relationship between the chain ordering and the entire swelling-critical and noncritical-seems to be independent of the sterol used and also of its concentration.

\section{Effect of sterols}

\section{Membrane swelling}

We see (Table I) that the more pronounced a sample shows the swelling effect [i.e., $d\left(T_{b}\right)-d\left(28{ }^{\circ} \mathrm{C}\right)$ ], the more $T_{c}$ moves towards the high-temperature end of the transition region $\Delta T_{m}^{\mathrm{wax}}$. The $10 \mathrm{~mol} \%$ cholesterol appears not to 
match this rule because the sterol concentration is obviously high enough to diminish the critical swelling [11]. Only the androsten samples actually have a $T_{c}$ inside the transition region $\Delta T_{m}^{\mathrm{wax}}$. Since the sterols, as basically hydrophobic entities incorporated into the lipid bilayer, interact in the first place with the aliphatic chains, they decrease the tilt angle of the ripple phase [25] upon incorporation. As a consequence they also increase the headgroup hydration by increasing the headgroup-headgroup spacing [11], accompanied by (critical) fluctuations resulting in a possible softening of the bilayer which in turn could cause an increased water layer (cf. model I). One might argue that the sterols overpower the origin of the anomalous swelling, a PC property, by decreasing the chain and/or headgroup tilt. This is not the case since the swelling remains critical for smaller amounts of sterols as shown above. A measure of the impaired periodic chain ordering (due to the presence of the sterols) is the width of the chain reflection dependent on the sterol concentration during the main transition. The chain reflection widths (measured in detector channels) are 49 (no sterol), 52 (5 mol \% androsten), 58 (10 mol \% androsten), 56 (5 mol \% cholesterol), and 63 (10 mol \% cholesterol). Because the chains are bound to the headgroups, the disorder induced in the chains should be transferred to the headgroups as well. Apparently, the impaired chain ordering, due to the sterols, yields a larger exponent $\alpha$ indicating a weakened criticality (Table I) but is not a monotonic function of the sterol concentration. In other words, the impaired periodic chain ordering (width) mirrors the degree of criticality $\alpha$, but is not the origin of the anomalous swelling.

\section{Cholesterol versus androsten}

For the cholesterol samples our data do not confirm the mutual exclusion of critical behavior and chain ordering because $T_{b}$ falls into the transition range $\Delta T_{m}^{\mathrm{wax}}$. However, the tentative statement made above - that the chain ordering is not the origin of the critical behavior-remains valid, last but not least because increased chain ordering does break the criticality in the cholesterol samples, too, as evidenced by a $T_{b}$ within the transition range $\Delta T_{m}^{\mathrm{wax}}$ (cf. Table I). Thus, swelling in the presence of cholesterol can exist with limited periodicity in chain ordering. Possible explanations for the differing amount of chain ordering, required to break the critical behavior observed between the two sterols, are (a) a sterol-specific interaction with the lipid molecules and (b) an active domain-boundary seeking of sterols in the coexistence region. Sterols, as sensors, have revealed an imperfect coupling of the chain ordering processes with the layer dynamics, i.e., the swelling, pointing towards a phenomenon affecting the hydrophilic-hydrophobic interface. Since none of the four models excludes changes in the headgroup-headgroup interaction, we now discuss the effects of critical fluctuations and hydration on these interactions.

\section{DISCUSSION}

\section{A. Testing the four models}

In the remainder of this paper, we associate the experiments with the four models discussed earlier [2]. Because

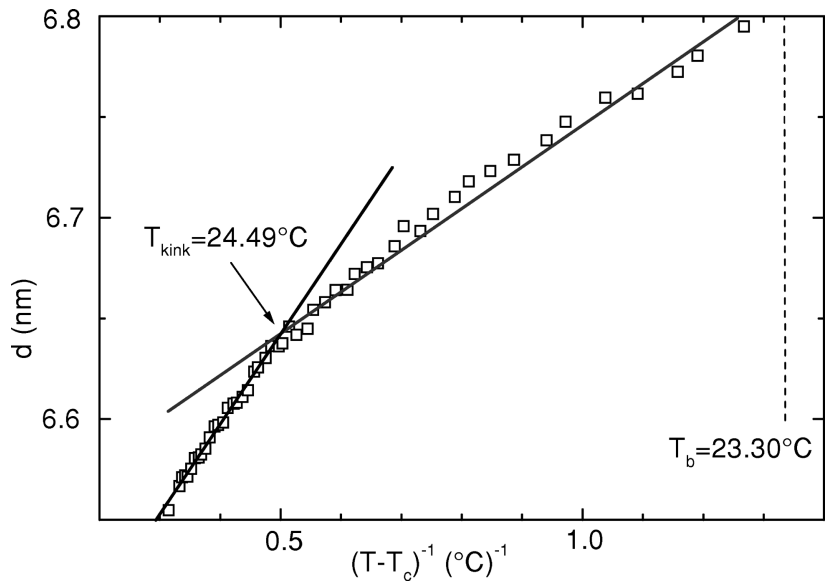

FIG. 6. Critical bilayer thickness (of vesicles of dimyristoylphosphatidylcholine, first cooling, scan rate $0.1{ }^{\circ} \mathrm{C} / \mathrm{min}, T_{c}$ $=22.55 \pm 0.05{ }^{\circ} \mathrm{C}$ ); a prediction [13] gives a straight line

undulations and fluctuations are basic to model I, we first discuss critical fluctuations and the correlation of fluctuations with swelling. The effect of chain ordering on the breakdown of criticality is connected with model II, and hydration is connected with models III and IV. The advent of a ripple phase appears to be connected with the main transition and precriticality. Finally, thermodynamic and nonequilibrium effects are discussed, and the importance of low scan rates is stressed.

\section{B. Critical fluctuations}

\section{Fluctuations in membranes}

Lipowsky and Leibler [14] predict, on the basis of Helfrich undulations, that the bilayer thickness $d$ should be linear in $1 /\left(T-T_{c}\right)$ (model I), i.e., $\alpha=1$. Our results (Table I) give exponents $\alpha$ that differ significantly from 1, whereas earlier experiments on lipid membranes [6] and other soft condensed matter systems [26] supported the predictions [14]. The magnitude of our precritical exponent $\alpha$ is reasonable, since an exponent of the order of 1 would yield a much larger swelling effect over the entire experimental critical regime of $28{ }^{\circ} \mathrm{C}$ down to $T_{b}$. Experimentally, even the largest amounts of critical swelling observed [Table I, $d\left(T_{b}\right)$ $\left.-d\left(28{ }^{\circ} \mathrm{C}\right)\right]$ do not reach a tenth of the unit cell size $d$ (of around $7 \mathrm{~nm}$ ). When plotting our data (Fig. 6) against $1 /(T$ $-T_{c}$ ), we observe clear deviations from the predicted linear behavior [14]; a single linear fit is not reasonable.

Thermal fluctuations trigger the main transition. To investigate the role of enhanced fluctuations (all models) on the swelling we consider the transition-related temperature derived from the layer reflections $T_{m}^{\mathrm{sax}}$, since it marks the temperature at the end of the swelling, critical plus noncritical. Our data (Table I) show that the addition of sterol shifts this transition temperature $T_{m}^{\mathrm{sax}}$. This means that only a partial periodic chain ordering is required to obtain the maximum $d$ spacing in the transition region. Even more, for the samples showing the strongest swelling effect yielding a local maximum in $d(T)$ (Fig. 2, 5 and $10 \mathrm{~mol} \%$ androsten), the continued ordering upon lowering temperature reduces $d(T)$ slightly. A more pronounced local maximum in the transition 

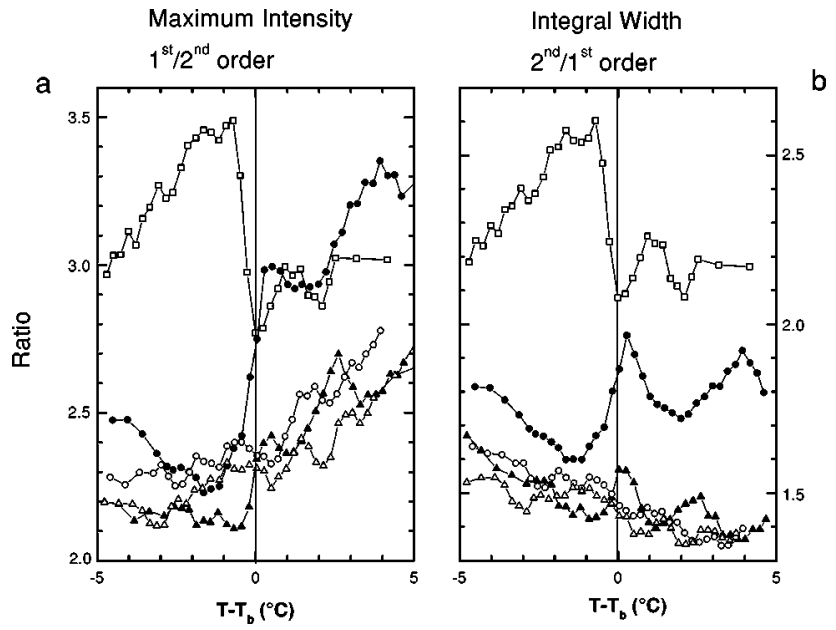

FIG. 7. Ratio of maximum intensities (a) and integral widths (b) of first- and second-order reflections as a function of temperature $T$ $\left(T_{b}\right.$ : breakdown temperature) for vesicles containing up to 10 mol \% sterol; symbols as in Fig. 2.

range is seen in the pure DMPC as well, but here it covers a broader temperature range compared to the two sterol samples. This peculiar relationship between chain ordering and $d$ spacing leading to the local maximum might be explained by the changing contributions of in-plane and out-ofplane fluctuations. Whereas the in-plane fluctuations affect the periodic ordering of the chains, the out-of-planefluctuations in first place deteriorate the periodicity of the layer order. The lower energetic in-plane fluctuations are expected to contribute more at the lower temperature end of the swelling when the interlayer spacing becomes larger and larger and neighboring membranes are basically decoupled. However, when the chains start to order due to increasing chain-chain coupling, these fluctuations will then be suppressed. In the theory of liquid crystals (lipid model membranes can be considered as such), in-plane fluctuations affect the bending modulus $K_{c}$ and the out-of-plane fluctuations affect the compression modulus $B$ [15]. Here, fluctuations broaden the width of the $\mathrm{x}$-ray reflections as a function of the order caused by the decreasing correlation length of the scattering units. Fluctuations, or defects of the first kind, also reduce the maximum intensity with increasing diffraction order [27].

\section{Fluctuations: Correlation with swelling}

The intensities of the $\mathrm{x}$-ray reflections give information about membrane fluctuations. Our data show [Fig. 7(a)] a decrease in the ratio of the maximum intensity of the firstorder to the second-order reflections, with increasing sterol concentration and with decreasing temperature, in the swelling region (of $28{ }^{\circ} \mathrm{C}$ down to $T_{b}$ ). Similarly, Fig. 7(b) shows that the ratios of the respective integral widths (integrated intensity/maximum intensity) have approximately the same $T$ variation for all sterol concentrations for $T>T_{b}$. In light of the theory of x-ray diffraction, the similarity of the dependence of both integral width and maximum intensity ratios on sterol concentration is to be interpreted as an in- crease of static defects [27], whereas the decreasing ratio of the maximum intensities with decreasing temperature when approaching $T_{b}$ (i.e., with increasing swelling) indicates a reduction of fluctuations (akin to a reduction in the DebyeWaller factor). Since the reduction is relatively stronger in the sterol samples than in the pure DMPC, it follows that sterols reduce fluctuations. In consequence, we have to exclude a significant fluctuation-driven contribution to the critical swelling, i.e., model I is not supported. [However, they increase the criticality of the system as expressed by $\alpha$ (Table I)]. The observed fluctuations are therefore not critical fluctuations and can be described in terms of the classical Ornstein-Zernicke theory [28]; in other words, the system is sufficiently far away from a critical point that a Landau-type theory is appropriate for the description of the fluctuations.

\section{Effect of chain ordering on the breakdown}

When comparing the breakdown temperature $T_{b}$ to the main transition zone $\Delta T_{m}^{\mathrm{wax}}$ (Table I), we observe that $T_{b}$ virtually coincides with the upper bound of $\Delta T_{m}^{\mathrm{wax}}$ for the pure lipid and the androsten samples on heating and cooling. For the cholesterol samples this is clearly not so (Table I): A certain degree of chain ordering appears to be permitted as $T_{b}$ moves into the transition zone $\Delta T_{m}^{\mathrm{wax}}$. This ordering effect must be related to the difference between the two sterols and their interaction with DMPC. The fact that for the pure lipid $T_{b}$ coincides with the upper bond of periodic chain ordering (given by $\Delta T_{m}^{\mathrm{wax}}$ ) is persuasive evidence for the following scenario. On the one hand, $T_{b}$ marks (by definition) the observed lower-temperature limit of the critical behavior [as defined by formula (2)] in the $L_{\alpha}$ phase. On the other hand, the upper bound of $\Delta T_{m}^{\mathrm{wax}}$ denotes the highertemperature limit of the periodic chain ordering of the ripple$P_{\beta^{\prime}}$ phase. Hence, on cooling, the onset of chain ordering breaks criticality (as is illustrated in Fig. 3, where the $T_{b}$ line intersects with the edge of a detectable WAX reflection). Therefore, changes in interactions within the hydrophobic membrane core, i.e., the steric repulsion and the van der Waals interaction between them, coupled to the chain ordering cannot directly control the critical swelling, so model II is not supported. (Models I, III, and IV are indifferent towards chain ordering effects.) Neither the steric repulsion nor the van der Waals interaction among the chains is expected to change drastically when entering the coexistence region, because both do not depend on the degree of periodic order. This is supported by the fact that one only observes critical swelling in PC but not, e.g., in phosphatidylethanolamine (PE) membranes. (Additional data taken on monomethylated and dimethylated PE do show the swelling.)

\section{Hydration}

The magnitude of the critical swelling $d\left(T_{b}\right)$ $-d\left(28{ }^{\circ} \mathrm{C}\right)$ is increased in the samples containing a small amount of sterol ( $\leqslant 10$ mol \%) (Fig. 2 and Table I), compared to that of the pure lipid. The adding of small amounts of sterol does not significantly decrease the area per headgroup [29], so that the difference in area per molecule in the neighboring phases remains large (about 15\%-20\% [1]). In turn we expect an increased amount of water in the head- 
group region possibly leading to a change in headgroup conformation, as is proposed by model IV. NMR measurements indicate that water molecules might penetrate past the hydrophilic headgroup towards the hydrophobic membrane core [16]. The changing hydration affects the hydrophilic/ hydrophobic interface. The dynamics of an interface is known to depend on the local curvature of the interface [30] and can behave critically under certain conditions [31]. The addition of $15 \%$ sterol does not provide appropriate conditions to observe critical swelling: Due to phase separation effects expected (a miscibility gap [1]) and the relatively low temperature in these experiments, no critical behavior can develop (cf. Fig. 2). Also, the diffraction technique is insensitive to short-range order. We have studies in progress on the miscibility gap [32]. A change of the hydration shell about the headgroup is thus a candidate for the critical swelling effect. We further remark that a decreased headgroup area upon adding sterol does increase the amount of swelling but reduces the criticality of the swelling (Table I). One is tempted to identify the kink in our results (Fig. 6), at around $24.55{ }^{\circ} \mathrm{C}$, as the boundary of a critical hydration dominance. (The changes in slope in Fig. 6 may be interpreted as a critical slowing down [33].) Lipowsky and Leibler imposed a hard confinement scenario in their derivation [14], which is perhaps not valid here [34], where a change in environment can be attributed to a change in hydration. Also, NMR data $[16]$ indicate a change in hydration at about this temperature. Moreover, dynamical scaling can break down despite the persistence of interfacial undulations [35]. To clarify the difference between the critical behavior of a changing headgroup hydration (model IV) and bilayer thickening (model II), we point out that the hydration depends on the headgroup environment whereas the bilayer thickening in model II is only a property of the chain conformation. The headgroup environment and the hydrophobic chains are coupled, and thus headgroup and hydrocarbon chains could be considered as an integral entity (model II). The differences between the two models are (a) the coupling is not stiff, so the behavior of chains and heads can be different and might not be mapped directly onto each other, and (b) chain-chain interactions involve lipids only, whereas hydration dynamics requires solvent (here water) and lipid molecules. These differences could give a clue why a lipid system can possess only precritical behavior, but not critical behavior.

When entering the coexistence region $\Delta T_{m}^{\mathrm{wax}}$, the headgroups feel the ordering of the coupled chains. As shown above, the chain ordering correlates with the breakdown of the critical swelling and hence breaks a critical interface dynamics. In response, the system finds a new (metastable) equilibrium position forming the ripple structure. It has been suggested that the swelling of the hydration shell at a hydrophobic/hydrophilic interface is a quite general phenomenon [36]. Also, osmotic pressure experiments on various PCs seem to show a critical dependence of the $d$ spacing on the osmotic pressure [34].

\section{SUMMARY AND CONCLUSION}

We demonstrated that the anomalous swelling in phosphatidylcholine membranes, of 14 carbons chain length, is precritical. By analyzing the nonlinear $d(T)$ behavior-in terms of the theory of critical phenomena-observed for the pure lipid and various sterol concentrations in the neighborhood of $T_{c}$, we were able to link the anomalous swelling to a changing headgroup hydration.

We propose that the formation of the ripple phase is the counterpart on the low-temperature side of the transition to the anomalous swelling on the high-temperature side. It is suggested that the ripple phase and the anomalous swelling are caused by the hydration dynamics of the headgroups.

We have found no evidence of critical entropic bilayer undulations quoted in model I. Model II suggests a critical thickening of the bilayer. The increase in the conformational ordering of the chains in the fluid phase, as they approach the coexistence region, is not known to obey critical dynamics.

Our sterol experiments show that adding small amounts of sterol does not break-but reduces-the criticality of the system, which seems to be linked to the larger headgroup area at the interface. This seems to be in agreement with model III, suggesting that a decreased interfacial surface increases the hydration force [37], however a decrease in interfacial area over the region of anomalous swelling cannot be supported by our data. This suggests that the hydration force does not change in a critical manner. The mechanism proposed by model IV, a conformational change of the headgroups due to changing hydration, is supported by our experiments and can explain the critical behavior. However, the model does not explain the amount of swelling [2].

From our data the following picture emerges: the changing hydration of the headgroups is responsible for the critical behavior and indirectly coupled chain ordering dynamics provides the full magnitude of the swelling. Further, the similarity of the transition-related temperatures determined from the SAX and WAX reflections (Table I) is evidence for a relationship of the conformational chain dynamics to the systems' transition from the fluid to the ripple phase, but not for the transition from the critical to the coexistence region, which is found to be related to the long-range periodic chain ordering dynamics.

Whereas DMPCholine exhibits critical swelling, DMPEthanolamine does not [38]. The much larger polar choline headgroup, the only structural difference between both lipids, is easy to hydrate. The existence of lattice critical swelling may well be connected with this difference in changing the hydration, and with the differences observed, between DMPC and DMPE, in differential scanning calorimetry [39] of the main transition.

We emphasize that our precritical exponents describe the swelling behavior over the entire temperature range (where swelling is observed, in all of our samples) supporting the subtle interface dynamics picture. However, a necessary requirement for an ab initio theoretical description is the inclusion of the formation of the ripple phase. This work suggests that the critical behavior is caused by the hydration dynamics.

\section{ACKNOWLEDGMENTS}

L.F. and G.R. thank NATO for Grant No. CRG 970225. L.F. is indebted to Michael A. Singer, of Queen's University, for discussions. Support of F.R. through the German BMBF through Grant No. 03-SA4TU2-5 is gratefully acknowledged. 
[1] E. Sackmann, in Structure and Dynamics of Membranes, edited by R. Lipowsky and E. Sackmann (North-Holland, Amsterdam, 1995), Chap. 5.

[2] J.F. Nagle, in Phase Transitions in Complex Fluids, edited by P. Toledano and A.M.F. Neto (World Scientific, Singapore, 1998).

[3] L. Finegold and M.A. Singer, in Cholesterol in Membrane Models, edited by L. Finegold (CRC Press, Boca Raton, 1993).

[4] R. Zhang, W. Sun, S. Tristram-Nagle, R.L. Headrick, R.M. Suter, and J.F. Nagle, Phys. Rev. Lett. 74, 2832 (1995).

[5] F.Y. Chen, W.C. Hung, and H.W. Huang, Phys. Rev. Lett. 79, 4026 (1997).

[6] J. Lemmich, K. Mortensen, J.H. Ipsen, T. Hønger, R. Bauer, and O.G. Mouritsen, Phys. Rev. Lett. 75, 3958 (1995).

[7] M.H. Hawton and J.W. Doane, Biophys. J. 52, 401 (1987).

[8] S. Mitaku, T. Jippo, and R. Kataoka, Biophys. J. 42, 137 (1983).

[9] S.M. Gruner and E. Shyamsunder, Ann. (N.Y.) Acad. Sci. 625, 685 (1991).

[10] M. Bloom, E. Evans, and O.G. Mouritsen, Q. Rev. Biophys. 24, 293 (1991).

[11] J. Lemmich, K. Mortensen, J.H. Ipsen, T. Hønger, R. Bauer, and O.G. Mouritsen, Eur. Biophys. J. 25, 293 (1997).

[12] W. Helfrich, J. Phys. (Paris) 47, 321 (1986); Z. Naturforsch. A 33, 305 (1978).

[13] R. Lipowsky, Europhys. Lett. 7, 255 (1988).

[14] R. Lipowsky and S. Leibler, Phys. Rev. Lett. 56, 2541 (1986).

[15] P.G. de Gennes, The Physics of Liquid Crystals (Clarendon Press, Oxford, 1974).

[16] K. Gawrisch, in Phase Transitions in Complex Fluids (Ref. [2]).

[17] S.E. Rankin, G.H. Addona, M.A. Kloczewiak, B. Bugge, and K.W. Miller, Biophys. J. 73, 2446 (1997).

[18] R. Varma and S. Mayor, Nature (London) 394, 798 (1998).

[19] G. Rapp, Acta Phys. Pol. A 82, 103 (1992).

[20] G. Rapp, A. Gabriel, M. Dosière, and M.H.J. Koch, Nucl. Instrum. Methods Phys. Res. A 357, 178 (1995).

[21] S. Matuoka, S. Kato, and I. Hatta, Biophys. J. 67, 728 (1994).

[22] J.J. Binney, N.J. Dorwick, A.J. Fisher, and M.E.J. Newman,
Theory of Critical Phenomena (Clarendon Press, Oxford, 1992), p. 5.

[23] H.E. Stanley, Introduction to Phase Transitions and Critical Phenomena (Clarendon Press, Oxford, 1971), p. 40.

[24] P.C. Hohenberg and B.I. Halperin, Rev. Mod. Phys. 49, 435 (1977); 49, 474ff (1977).

[25] D. Needham, T.J. McIntosh, and E. Evans, Biochemistry 27, 4668 (1988).

[26] C.R. Safinya, E.B. Sirota, D. Roux, and G.S. Smith, Phys. Rev. Lett. 62, 1134 (1989).

[27] A. Guinier, Principles of X-ray Diffraction (W.H. Freeman, San Francisco, 1963), Chap. 9.

[28] L.D. Landau and E.M. Lifschitz, Statistical Physics, Course of Theoretical Physics Vol. 5 (Addison-Wesley, Reading, 1958).

[29] M.J. Zuckermann, J.H. Ipsen, and O.G. Mouritsen, in Cholesterol in Membrane Models, edited by L. Finegold (CRC Press, Boca Raton, 1993).

[30] T. Taniguchi, Phys. Rev. Lett. 76, 4444 (1996).

[31] P. Meakin, Fractals, Scaling and Growth Far from Equilibrium (Cambridge University Press, Cambridge, 1998).

[32] G. Rapp, L. Finegold, and F. Richter, Biophys. J. 76, A63 (1999).

[33] A. Aharony, in Phase Transitions and Critical Phenomena, edited by C. Domb and M.S. Green (Academic Press, London, 1976), Vol. 6, Chap. 5.

[34] H.I. Petrache, N. Gouliaev, and J.F. Nagle, Phys. Rev. E 57, 7014 (1998).

[35] O. Schönborn and R.C. Desai, Eur. J. Phys. (to be published); J. Stat. Phys. (to be published).

[36] D.I. Svergun, S. Richard, M.H.J. Koch, Z. Sayers, S. Kupin, and G. Zaccai, Proc. Natl. Acad. Sci. USA 95, 2267 (1998).

[37] T.J. McIntosh and S.A. Simon, Biochemistry 32, 8374 (1993).

[38] M. Rappolt and G. Rapp, Ber. Bunsenges. Phys. Chem. 100, 1153 (1996).

[39] A. Blume, in Physical Properties of Biological Membranes and their Functional Implications, edited by C. Hidalgo (Plenum, London, 1988).

[40] J.F. Nagle, H. Petrache, N. Gouliaev, S. Tristram-Nagle, Y. Liu, R.M. Suter, and K. Glawrisch, Phys. Rev. E 58, 7769 (1998). 